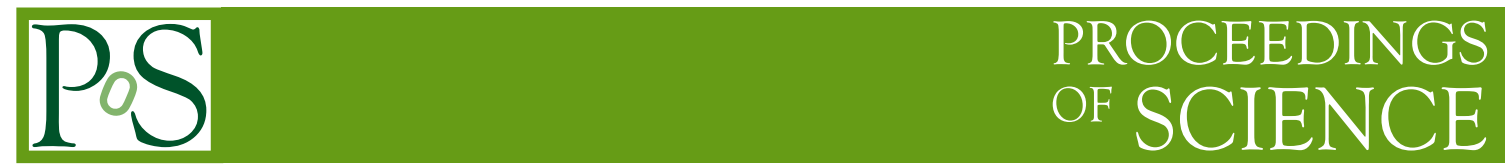

\title{
Measurement of the CKM angle $\gamma$ at LHCb
}

\author{
Sneha Malde*, on behalf of the LHCb Collaboration \\ University of Oxford \\ E-mail: sneha.malde@physics.ox.ac.uk
}

The CKM angle $\gamma$ is the least well known angle of the unitarity triangle, and the only one easily accessible at tree level. The ultimate goal of degree level precision requires exploitation of all possible channels and techniques. Presented here are some of the latest results on the CKM angle $\gamma$ from the LHCb experiment. Included are measurements of $C P$ violation using the $B^{ \pm} \rightarrow$ $D K^{ \pm}$decays and a variety of different $D$ decay modes, and a measurement $\gamma$ from $B^{0} \rightarrow D K^{* 0}$ where the $D$ decays to either $K_{s}^{0} \pi^{+} \pi^{-}$or $K_{s}^{0} K^{+} K^{-}$. A combination of these and other $\gamma$-related measurements is also presented.

38th International Conference on High Energy Physics 3-10 August 2016

Chicago, USA

${ }^{*}$ Speaker. 


\section{Introduction}

Of the three angles that make up the CKM unitarity triangle, the least well known remains $\gamma$ [1]. A precise measurement of this quantity is highly desirable. Assuming the absence of new physics within tree-level decays, the direct measurement of $\gamma$ forms a Standard Model benchmark. Through the use of all other CKM observables an indirect determination of $\gamma$ can be made which is driven by the measurement of the angle $\beta$ and the ratio of $\Delta\left(m_{s}\right) / \Delta\left(m_{d}\right)$, both of which are loop-level processes and hence their values could be altered by new physics particles participating within the virtual loops [2]. The CKMfitter group determines that the combination of all direct measurements of $\gamma=\left(73.2_{-7.0}^{+6.3}\right)^{\circ}$, while an indirect determination that excludes the direct measurements leads to $\gamma=\left(66.85_{-3.44}^{+0.94}\right)^{\circ}$ [1]. It is clear that a reduction in the uncertainty associated with the direct measurements is required to enable the chance to observe a discrepancy between these two values of $\gamma$.

The results presented in these proceedings demonstrate some of the variety in the decay modes and techniques that can be used to determine $\gamma$ at LHCb. The results are based on the full Run-1 dataset which was collected in 2011 and 2012, corresponding to $3 \mathrm{fb}^{-} 1$. The decay modes useful for studies of the CKM angle $\gamma$ are fully hadronic and therefore benefit from a number of features of the LHCb experiment including the low-level hadronic trigger, the excellent vertex and momentum resolution and the good separation between pions and kaons due to the RICH detectors.

\section{2. $C P$ violation in $B^{ \pm} \rightarrow D K^{ \pm}$decays}

Sensitivity to $\gamma$ arises from the interference of $b \rightarrow c$ and $b \rightarrow u$ quark transitions. The most used decays are $B^{+} \rightarrow{\overline{D^{0}}}^{+}$(favoured) and $B^{+} \rightarrow D^{0} K^{+}$(suppressed) ${ }^{1}$. For interference to occur, the $\bar{D}^{0}$ and $D^{0}$ must decay to a final state accessible to both. The overall decay in this situation is referred to as $B^{+} \rightarrow D K^{+}$where $D$ represents a superposition of $D^{0}$ and $\overline{D^{0}}$. A number of $D$ modes can be used. The asymmetry in the observed number of $B^{-}$and $B^{+}$decays in corresponding final states is related to three physics parameters that are common across all $B^{+} \rightarrow D K^{+}$analyses. They are $r_{B}$, the ratio of the suppressed and favoured $B$ decay amplitudes, $\delta_{B}$ the strong-phase difference between the suppressed and favoured $B$ decay amplitudes, and $\gamma$, the parameter of primary interest. Depending on the $D$ decay, further hadronic parameters relating to the decay of the $D$ also feature in the equations that relate the observed rates of decay to $\gamma$.

A very sensitive $D$ decay mode is $D^{0} \rightarrow K^{+} \pi^{-}$which is doubly-Cabibbo-suppressed. The use of this decay mode allows the two interfering amplitudes to be of similar size, which enhances the sensitivity to $\gamma$, and is commonly referred to as an 'ADS' decay mode [3] after the theorists who first proposed it. The asymmetry between $B^{-}$and $B^{+}$is related to $\gamma$ and other parameters via

$$
\frac{\Gamma\left(B^{-}\right)-\Gamma\left(B^{+}\right)}{\Gamma\left(B^{-}\right)+\Gamma\left(B^{+}\right)}=\frac{2 r_{B} r_{D} \sin \left(\delta_{B}+\delta_{D}\right) \sin (\gamma)}{r_{B}^{2}+r_{D}^{2}+2 r_{B} r_{D} \cos \left(\delta_{B}+\delta_{D}\right) \cos (\gamma)}
$$

where $r_{D}$ and $\delta_{D}$ are analogous parameters for the $D$ decay and are measured through studies of $D$ mixing. The invariant mass distribution for this decay is shown in Fig. 1. The total signal yield is 550 events, There is a clear enhancement of the yield of the $B^{+}$decay in comparison to the $B^{-}$

\footnotetext{
${ }^{1}$ Charge conjugation is implied throughout
} 
decay. This decay has only been observed at $\mathrm{LHCb}$, and the $C P$ violation is observed at a level of $8 \sigma[4]$.
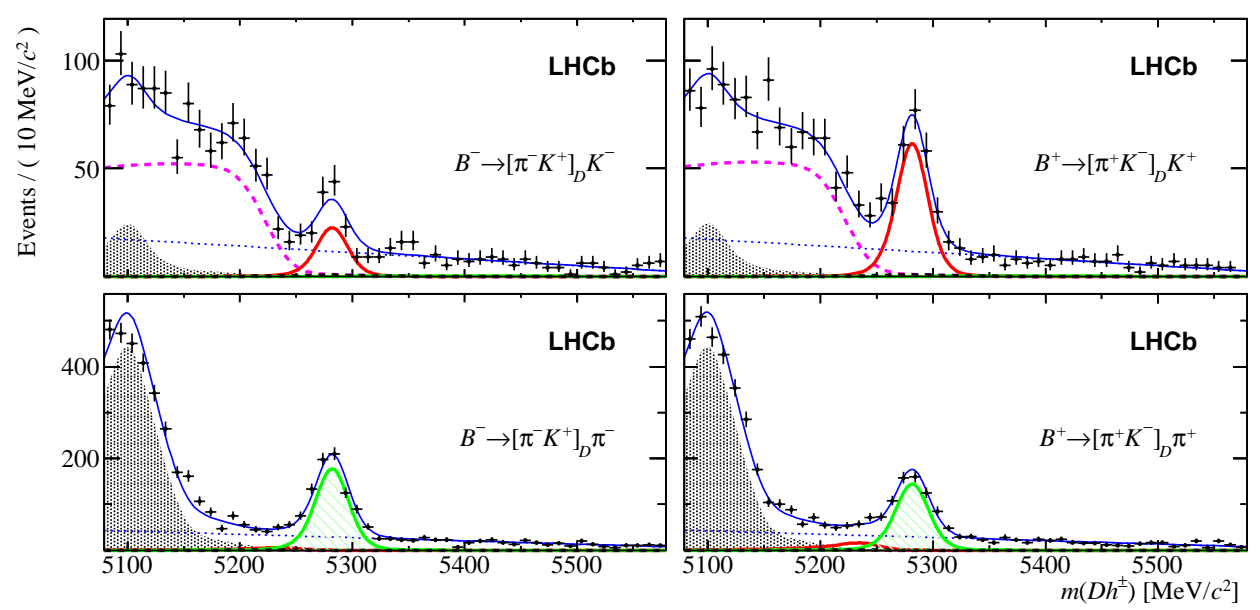

Figure 1: Invariant mass distributions of selected $B^{ \pm} \rightarrow\left[K^{\mp} \pi^{ \pm}\right]_{D} h^{ \pm}$candidates, separated by charge, with $B^{-}\left(B^{+}\right)$candidates on the left (right). The top plots contain the $B^{ \pm} \rightarrow D K^{ \pm}$candidate sample. The bottom row contains the $B^{ \pm} \rightarrow D \pi^{ \pm}$sample. The red (thick, open) and green (hatched-area) curves represent the $B \rightarrow D K$ and $B \rightarrow D \pi$ signals. The shaded part indicates partially reconstructed decays, the dotted line, where visible, shows the combinatorial component, and the total PDF is drawn as a thin blue line.

Another family of $D$ decay modes that can be used are self-conjugate decays such as $D \rightarrow$ $\pi^{+} \pi^{-} \pi^{+} \pi^{-}$. These are referred to as quasi-GLW [5] decay modes (where the quasi is dropped in the case of a $C P$ eigenstate [6]). In this case the asymmetry is related to

$$
\frac{\Gamma\left(B^{-}\right)-\Gamma\left(B^{+}\right)}{\Gamma\left(B^{-}\right)+\Gamma\left(B^{+}\right)}=\frac{2 r_{B}\left(2 F_{+}-1\right) \sin \left(\delta_{B}\right) \sin (\gamma)}{1+r_{B}^{2}+2 r_{B}\left(2 F_{+}-1\right) \cos \left(\delta_{B}\right) \cos (\gamma)}
$$

where $F_{+}$is a hadronic parameter of the $D$ decay which represents the $C P$-even content of the decay. For a $C P$ even (odd) eigenstate $F_{+}$would be $1(0)$. In the case of $D \rightarrow \pi^{+} \pi^{-} \pi^{+} \pi^{-}, F_{+}$ has been measured using data from the CLEO-c and is determined to be $0.737 \pm 0.028$ [7]. The asymmetry is shown in Fig 2. A small hint of asymmetry is seen and this is the first time this decay mode has been used to provide sensitivity to $\gamma$. This is only possible due to the external information on $F_{+}$. In both Figs. 1 and 2 the invariant mass distributions for the related decay in $B^{ \pm} \rightarrow D \pi^{ \pm}$are also shown. $C P$ violation effects are expected to be less noticeable here given that the ratio of $B$ decay amplitudes, $r_{B}^{\pi}$, is expected to be about an order of magnitude smaller than $B^{ \pm} \rightarrow D K^{ \pm}$. Nonetheless the combination of asymmetries in $B^{+} \rightarrow D \pi^{+}$decays using the decay modes $D^{0} \rightarrow K^{+} K^{-}, \pi^{+} \pi^{-}, K^{+} \pi^{-}, \pi^{+} \pi^{-} \pi^{+} \pi^{-}$and $K^{+} \pi^{-} \pi^{+} \pi^{-}$leads to evidence of $C P$ violation at the level of $3.9 \sigma$.

\section{3. $C P$ violation in the decay $B^{0} \rightarrow D K^{* 0}$}

Studies of $\gamma$ are not restricted to $B^{+} \rightarrow D K^{+}$. The $B^{0} \rightarrow D K^{* 0}$ decay is expected to be promising as in this case the ratio of amplitudes of the favoured and suppressed $B$ decays, $r_{B^{0}}$ is expected 


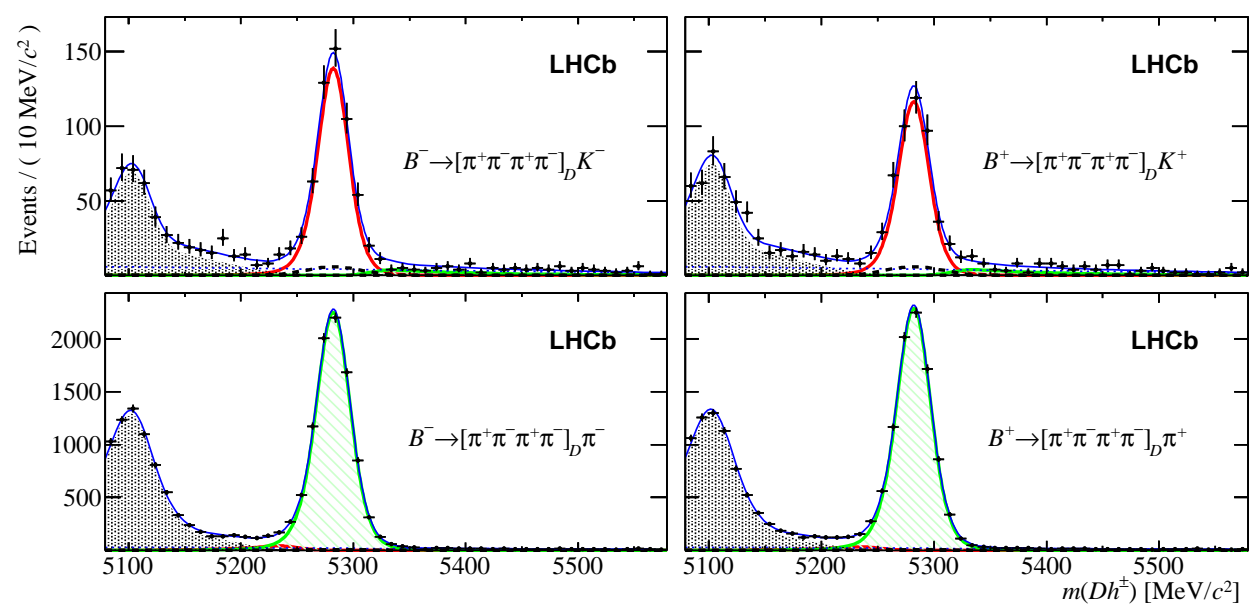

Figure 2: Invariant mass distributions of selected $B^{ \pm} \rightarrow\left[\pi^{+} \pi^{-} \pi^{+} \pi^{-}\right]_{D} h^{ \pm}$candidates, separated by charge, with $B^{-}\left(B^{+}\right)$candidates on the left (right). The top plots contain the $B^{ \pm} \rightarrow D K^{ \pm}$candidate sample. The bottom row contains the $B^{ \pm} \rightarrow D \pi^{ \pm}$sample. The red (thick, open) and green (hatched-area) curves represent the $B \rightarrow D K$ and $B \rightarrow D \pi$ signals. The shaded part indicates partially reconstructed decays, the dotted line, where visible, shows the combinatorial component, and the total PDF is drawn as a thin blue line.

to be around 0.3, and hence the enhanced interference will compensate for the lower branching fraction of this decay in comparison to $B^{ \pm} \rightarrow D K^{ \pm}$. Despite this being a neutral $B$ meson there is no need for time dependence or flavour tagging as the charge of the Kaon from the $K^{* 0} \rightarrow K^{+} \pi^{-}$ tags the flavour of the $B$ at the point of decay. The $D$ decay modes used in a recent analysis are $D \rightarrow K_{S}^{0} \pi^{+} \pi^{-}$and $D \rightarrow K_{S}^{0} K^{+} K^{-}$(referred to as 'GGSZ' modes [9]). While these are selfconjugate modes it is not prudent to analyse them in a quasi-GLW style way since $F_{+} \sim 0.5$. Hence there is expected to be very little asymmetry in the overall $B^{0}$ and $\bar{B}^{0}$ yields. However in this case the rich resonance structure of the three-body $D$ decay can be harnessed to provide the sensitivity to $\gamma$. In this analysis the Dalitz plot is divided into regions. The signal yield in each Dalitz plot bin is related to $\gamma, r_{B^{0}}, \delta_{B^{0}}$ and the charm hadronic and strong-phase parameters relating to the average $D$ decay amplitudes in those bins. The charm hadronic and strong-phase parameters are determined from an analysis using CLEO-c data that exploits the quantum correlation in $\psi(3770) \rightarrow D D$ decays, and therefore has direct access to the strong-phases of the decay [8]. This is an advantage as the subsequent measurement of $\gamma$ has well-defined uncertainties and is not subject to uncertainties from phases determined in amplitude models of the $D$ decay which can be difficult to assign. The interference in this $B^{0} \rightarrow D K^{* 0}$ is rich, and allows for a standalone determination of $\gamma$ $=(71 \pm 20)^{\circ}$ [10] with a further solution at $(71+180)^{\circ}$. The 1 and $2 \sigma$ contours of $\gamma$ vs $r_{B^{0}}$ and $\gamma$ vs $\delta_{B^{0}}$ are depicted in Fig. 3. A model-dependent analysis has also been performed at LHCb where the hadronic parameters of the $D$ decay are determined from an amplitude analysis. The results of the two analyses are compatible [11].

\section{Combination of all analyses}

As seen in Eqs 2.1 and 2.2 the relation between the laboratory observables and the physics 

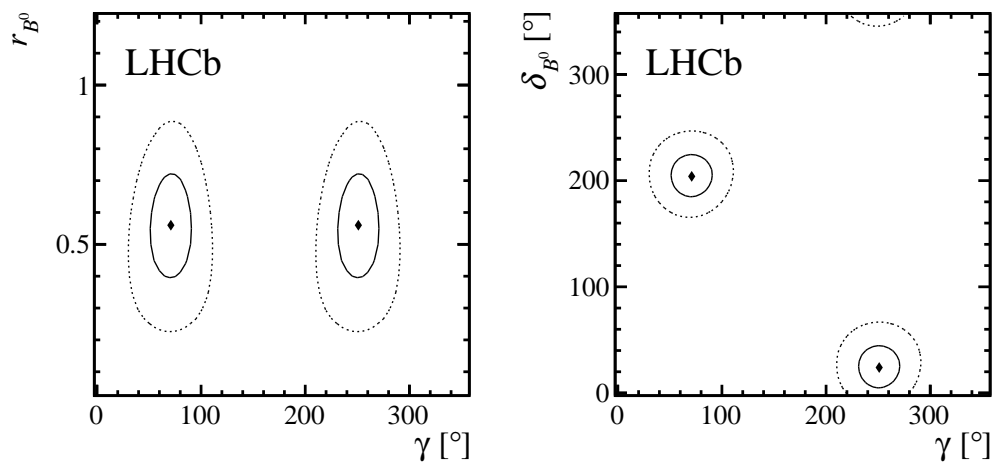

Figure 3: The three-dimensional confidence volumes projected onto the $\left(\gamma, r_{B^{0}}\right)$ and $\left(\gamma, \delta_{B^{0}}\right)$ planes. The confidence levels correspond to $68.3 \%$ and $95.5 \%$ confidence levels when projected onto one dimension and are denoted by solid and dotted contours, respectively. The diamonds mark the central values.

parameter of interest can contain complex trigonometrical relations which can lead to multiple solutions. The interplay between different $B^{ \pm} \rightarrow D K^{ \pm}$analyses is shown in Fig 4. The ADS and (quasi)-GLW decay modes lead to 4 separate solutions with small uncertainty (blue, beige). Conversely the 'GGSZ' decay mode analysis leads to a single solution albeit with larger uncertainty (pink). Hence the power is in the combination (red). The $B$ modes included are $B^{ \pm} \rightarrow D K^{ \pm}$, $B^{ \pm} \rightarrow D K^{ \pm} \pi^{ \pm} \pi^{\mp}, B^{0} \rightarrow D K^{* 0}, B^{0} \rightarrow D K^{+} \pi^{-}$, and $B_{s} \rightarrow D_{s} K$ with a range of subsequent $D$ decays. In total there are 71 observables and 32 parameters involved with a number of external inputs to describe some of the $D$ decays. Frequentist and Bayesian combinations are performed with the results in good agreement. The combination of these results yields $\gamma=\left(70.9_{-8.5}^{+7.1}\right)^{\circ}$ [12] and the contribution from different $B$ decays is shown in Fig $4^{2}$. This level of sensitivity meets the LHCb Run 1 target, is approximately a factor of two better than either of the B factory combinations and is $20 \%$ more precise than the previous LHCb combination from 2014.

\section{Outlook}

The LHCb experiment continues to analyse a wider variety of $B$ and $D$ decays to improve the precision on the CKM angle $\gamma$. Data from Run-2 is now being analysed and plans for the LHCb upgrade should allow for degree level precision within 15 years [13]. To probe for new physics it is evident that precision of the order of a degree will be required, and this remains one of the main goals of the LHCb experiment. The results from Run-1 have provided a significant improvement in our knowledge of the parameter $\gamma$ and the LHCb experiment demonstrates that it remains on target to reach degree level precision with the data from the LHCb upgrade.

\section{References}

[1] [CKMFitter group] J. Charles et al., Updated results and plots available at : http://ckmfitter.in2p3.fr, Eur. Phys. J C41 2005 1, [hep-ph/0 406184 ]

\footnotetext{
${ }^{2}$ Since the conference these preliminary results and plots regarding the combination have been updated. Information can be found in LHCb-PAPER-2016-032, which will be submitted to JHEP in November 2016.
} 

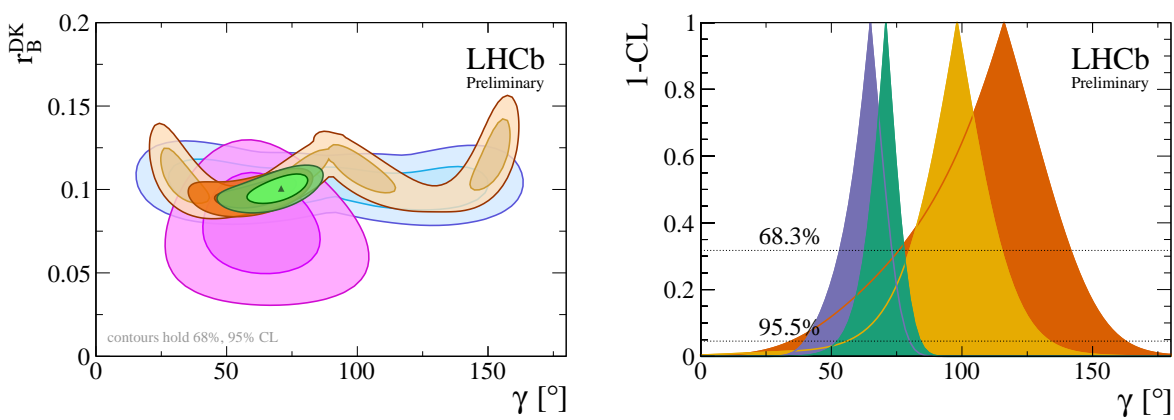

Figure 4: Left: Two-dimensional distributions of $\gamma$ vs. $r_{B}^{D K}$ for various sub-combinations. These are (blue) $B^{+} \rightarrow D K^{+}, D \rightarrow h \pi \pi \pi / h h^{\prime} \pi^{0}$, (beige) $B^{+} \rightarrow D K^{+}, D \rightarrow h h$, (pink) $B^{+} \rightarrow D K^{+}, D \rightarrow K_{s}^{0} h h$, (red) all $B^{+}$ modes and (green) the full combination, where $h$ is either a kaon or pion. Right:1-CL plots for combinations split by the initial $B$ meson flavour as follows: (orange) $B_{s}^{0}$, (yellow) $B^{0}$, (blue) $B^{+}$and (green) the full combination.

[2] M. Blanke and A. J. Buras, Universal Unitarity Triangle 2016 and the tension between $\Delta M_{s, d}$ and $\varepsilon_{K}$ in CMFV models, Eur. Phys. J C76 2016 197, [arXiv: 1602 . 04020]

[3] D. Atwood, I. Dunietz and A. Soni, Enhanced $C P$ violation with $B \rightarrow K D(\bar{D})$ modes and extraction of the CKM angle $\gamma$, Phys. Rev. Lett. 781997 3257, [hep=ph/9612433]

[4] [LHCb collaboration] R. Aaij et al., Measurement of $C P$ observables in $B \rightarrow D K$ and $B \rightarrow D \pi$ with two and four body decays, Phys. Lett. B760 2016117

[5] M. Nayak et al., First determination of the CP content of $D \rightarrow \pi \pi \pi^{0}$ and $D \rightarrow K K \pi^{0}$, Phys. Lett B740 2015 1, [arXiv: 1410.3964 ]

[6] M. Gronau and D. Wyler, On determining a weak phase from CP asymmetries in charged B decays, Phys. Lett. B265 1991172

[7] S. Malde et al., First determination of the CP content of $D \rightarrow \pi \pi \pi \pi$ and updated determination of the $C P$ contents of $D \rightarrow \pi \pi \pi^{0}$ and $D \rightarrow K K \pi^{0}$, Phys. Lett. B747 2015 9, [arXiv: 1504.05878 ]

[8] [CLEO collaboration] J. Libby et al. Model-independent determination of the strong=phase difference between $D^{0}$ and $\bar{D}^{0} \rightarrow K_{S, L} h^{+} h^{-}(h=\pi, K)$ and its impact on the measurement of the CKM angle $\gamma / \phi_{3}$, Phys. Rev. D82 2010112006 [arXiv: 1010.2817 ]

[9] A. Giri, Y. Grossman, A. Soffer and J. Zupan, Determining gamma using $B \rightarrow D K$ with multibody D decays Phys. Rev. D 682003 054018, [arXiv: hep-ph/0303187]

[10] [LHCb collaboration], R. Aaij et al., Model-independent measurement of the CKM angle $\gamma$ using $B^{0} \rightarrow D K^{* 0}$ with $D \rightarrow K_{s} \pi \pi$ and $D \rightarrow K_{S} K K$, JHEP 062016 131, [arXiv. 1604.01525 ]

[11] [LHCb collaboration], R. Aaij et al., Measurement of the CKM angle $\gamma$ using $B^{0} \rightarrow D K^{* 0}$ with $D \rightarrow K_{s} \pi \pi$ decays, JHEP $\mathbf{0 8} 2016$ 137, [arXiv. 1605.0182]

[12] LHCb collaboration, Measurement of the CKM angle $\gamma$ from a combination of $B \rightarrow D K$ analyses, LHCb-CONF-2016-001

[13] LHCb collaboration et al., Implications of LHCb measurements and future prospects, Eur. Phys. J C73 20132373 